\title{
RELAÇÃO TERAPEUTA PACIENTE E DIMENSÕES DA HUMANIZAÇÃO EM REABILITAÇÃO ORTOPÉDICA
}

\section{Eloisa Cereda Oliveira}

Bacharel em Fisioterapia pelo Centro Universitário Fundação Assis Gurgacz - FAG, Brasil.

\section{Antuani Rafael Baptistella}

Doutor em Oncologia. Docente do Programa de Mestrado em Biociências e Saúde. Fisioterapeuta, Brasil.

\section{Marcelo Taglietti}

Doutor em Educação Física. Docente do Departamento de Fisioterapia. Fisioterapeuta, Brasil.

\begin{abstract}
RESUMO: Para promover a qualidade de vida aos pacientes atendidos em um programa de reabilitação, há a necessidade e obrigação de humanizar o atendimento. O fisioterapeuta exerce um papel fundamental na reabilitação dos pacientes com sequelas ortopédicas, e, além de qualidade técnica, deve prezar pela qualidade relacional de sua assistência. avaliar a relação terapeuta paciente, investigando se há humanização do atendimento fisioterapêutico na especialidade de ortopedia. Estudo de corte transversal, no qual foi aplicado um questionário adaptado avaliativo individual, contendo 15 questões fechadas em nove domínios, em uma amostra de 59 indivíduos, os quais receberam atendimento na especialidade de fisioterapia ortopédica. Os dados foram analisados e apresentados em frequência e porcentagem. $\mathrm{O}$ atendimento fisioterapêutico foi caracterizado como humanizado, obtendo-se resposta com $100 \%$ em todos os domínios do questionário, nos quesitos que dizem respeito à relação terapeutapaciente. A assistência fisioterápica, na especialidade de ortopedia, foi caracterizada como humanizada pelos pacientes.
\end{abstract}

PALAVRAS-CHAVE: Fisioterapia; Ortopedia; Humanização da assistência; Promoção da saúde.

\section{THERAPIST-PATIENT RELATIONSHIP AND HUMANIZATION IN ORTHOPEDIC REHABILITATION}

ABSTRACT: Care should be humanized to promote the life quality of patients attended in a rehabilitation program. The physiotherapist has an important role in the rehabilitation of patients with orthopedic sequels. Besides technical quality, physiotherapists should appreciate the quality of their relationship. To evaluate the therapist-patient relationship by investigating whether humanization exists in physiotherapist attendance, especially in orthopedics. Transversal research applied a questionnaire adapted for individual evaluation, with 15 questions within nine dominions, in a sample of 59 agents receiving care in orthopedic physiotherapy. Data were analyzed, with frequency and percentages. Physiotherapeutic attendance was characterized as humanized, with one hundred percent response in all the domains of the questionnaire with regard to therapist-patient relationship. Physiotherapy, especially orthopedics, was characterized as humanized by patients.

KEY WORDS: Physiotherapy; Orthopedics; Humanization of aid; health promotion. 


\section{INTRODUÇÃO}

Humanizar refere-se à possibilidade de uma modificação cultural da gestão e das habilidades desenvolvidas nas instituições de saúde, tornando-se uma postura ética de respeito ao outro, de acolhimento do desconhecido, de respeito ao usuário, sendo entendido como um cidadão e não apenas como um consumidor de serviços de saúde. ${ }^{1}$

A partir da segunda metade do século XX, a qualidade dos serviços de saúde passou a ser referida e interrogada com mais ênfase, quando se iniciaram as pesquisas sobre a avaliação da assistência técnica em saúde, com destaque na qualidade do atendimento. ${ }^{2}$

Com isso, programas foram lançados no âmbito nacional para dar suporte aos profissionais da saúde nos quesitos de aprofundamento dos modelos de relação terapeuta paciente, bem como na humanização da assistência, destacando-se o programa HumanizaSUS, AprenderSUS, Programa Nacional de Humanização da Assistência Hospitalar e a Política Nacional de Humanização.

Medidas clínicas são em geral utilizadas para avaliação sobre o sucesso do tratamento no cuidado de pacientes, dentre elas, os questionários e outros instrumentos são amplamente utilizados por fisioterapeutas para medir o estado de saúde do paciente ou os efeitos do tratamento executado, embora sendo considerada de pouca valia, a satisfação do paciente é primordial no cuidado de pessoas.

Dentro da fisioterapia, apenas quatro estudos ${ }^{3-6}$ avaliaram a relação terapeuta paciente, todos no ambiente hospitalar e com serviços no âmbito público, onde as condutas mostraram-se humanizadas. Nenhuma pesquisa foi realizada em instituições ou serviços fora do meio hospitalar.

Através disso, o objetivo do estudo foi avaliar a relação terapeuta paciente em relação aos procedimentos fisioterápicos incluídos em seu processo de recuperação e investigar se há humanização do atendimento fisioterapêutico realizado em um Centro de Reabilitação na especialidade de ortopedia.

\section{METODOLOGIA}

Trata-se de um estudo transversal, realizado de fevereiro a setembro de 2017 , com pacientes que receberam alta no centro de reabilitação, na especialidade de ortopedia, pertencente as Clínicas FAG, a qual inclui atendimentos de caráter público e privado. A amostra foi definida por conveniência e incluíram 59 indivíduos maiores de 18 anos que corresponderam aos seguintes critérios de inclusão: histórico de receber atendimento fisioterápico no centro de reabilitação; ser lúcido e orientado, com capacidade de verbalização oral e/ ou escritas preservadas pela análise do entrevistador; quando menor de idade, acompanhado do pai ou responsável e; concordar em fazer parte do estudo. Foram excluídos aqueles que não receberam intervenção por fisioterapeutas e que não concordaram em participar da pesquisa.

O projeto foi analisado e aprovado pelo Comitê de Ética e Pesquisa do Centro Universitário Assis Gurgacz, sob parecer número 1.854 .209 e autorizado pelo local da pesquisa. Os pacientes foram assegurados de que seus dados e informações permaneceriam confidenciais e sigilosos, não permitindo sua identificação.

Os pesquisadores receberam informações diárias sobre as altas dos pacientes da especialidade de fisioterapia traumato-ortopédica pelos 11 fisioterapeutas atuantes, sendo a pesquisa realizada em forma de entrevista face a face, no consultório de avaliação, após esclarecimento e assinatura do Termo de Consentimento Livre e Esclarecido.

O questionário utilizado foi adaptado, seguindo os padróes estruturados e desenvolvido por Lopes e $\mathrm{Brito}^{3}$, composto por questões fechadas incluindo dados sociodemográficos, análise da relação fisioterapeuta-paciente e dos procedimentos adotados pelos fisioterapeutas, considerados de forma humanizada (positiva) ou desumanizada (negativa) (Apêndice I). Foi categorizada como "humanizada" quando representada por cinco ou mais respostas positivas na avaliação da relação fisioterapeuta-paciente, e 'desumanizada', por cinco ou mais respostas negativas na avaliação dessa relação. Assim colhidos, os dados foram transportados, tabulados e analisados pelo programa SPSS $^{\circledR}$ versão 
24.0, no qual se realizou sua distribuição de frequência, sendo apresentados em porcentagem, frequência bruta e relativa.

\section{RESULTADO}

De acordo com a Tabela I, observa-se que 55,9\% dos pacientes são do sexo feminino, 67,8\% têm faixa etária definida entre 18 e 59 anos, 57,6\% são casados, $37,3 \%$ não concluíram o ensino fundamental e $86,4 \%$ mantêm uma renda de zero a três salários mínimos.

Tabela 1. Características sociodemográficas

\begin{tabular}{|c|c|c|}
\hline & $\begin{array}{l}\mathbf{N}^{\circ} \text { ( frequên- } \\
\text { cia) }\end{array}$ & Porcentagem \\
\hline \multicolumn{3}{|l|}{ Sexo } \\
\hline Feminino & 33 & $55,9 \%$ \\
\hline Masculino & 26 & $44,1 \%$ \\
\hline \multicolumn{3}{|l|}{ Faixa etária } \\
\hline 18 a 59 anos & 40 & $67,8 \%$ \\
\hline$\geq 60$ anos & 19 & $32,2 \%$ \\
\hline \multicolumn{3}{|l|}{ Estado civil } \\
\hline Solteiro & 17 & $28,8 \%$ \\
\hline Casado & 34 & $57,6 \%$ \\
\hline Viúvo & 6 & $10,2 \%$ \\
\hline Divorciado & 2 & $3,4 \%$ \\
\hline \multicolumn{3}{|l|}{ Escolaridade } \\
\hline $\begin{array}{l}\text { Sem escolaridade } \\
\text { formal }\end{array}$ & 9 & $15,3 \%$ \\
\hline $\begin{array}{l}\text { Ensino fundamental } \\
\text { incompleto }\end{array}$ & 22 & $37,3 \%$ \\
\hline $\begin{array}{l}\text { Ensino fundamental } \\
\text { completo }\end{array}$ & 6 & $10,2 \%$ \\
\hline $\begin{array}{l}\text { Ensino médio } \\
\text { incompleto }\end{array}$ & 2 & $3,4 \%$ \\
\hline $\begin{array}{l}\text { Ensino médio } \\
\text { completo }\end{array}$ & 12 & $20,3 \%$ \\
\hline $\begin{array}{l}\text { Ensino superior } \\
\text { incompleto }\end{array}$ & 4 & $6,8 \%$ \\
\hline $\begin{array}{l}\text { Ensino superior } \\
\text { completo }\end{array}$ & 4 & $6,8 \%$ \\
\hline \multicolumn{3}{|l|}{ Renda (salários mínimos) } \\
\hline 0 a 3 salários & 51 & $86,4 \%$ \\
\hline >3 salários & 8 & $13,6 \%$ \\
\hline
\end{tabular}

$\mathrm{Na}$ avaliação da relação fisioterapeuta-paciente foram observados resultados positivos quanto ao atendimento fisioterapêutico em reabilitação, em que os pacientes concluíram que o atendimento foi realizado de maneira humanizada. As dimensões dignidade, comunicação, autonomia, confiabilidade, garantia, aspectos interpessoais, empatia, eficácia e receptividade obtiveram $100 \%$ de positividade nas respostas. Nenhuma das dimensões da relação fisioterapeuta-paciente apresentaram resultado negativo. Portanto, a conduta fisioterapêutica prestada no centro de referência foi concluída com 100\% de aprovação pelos indivíduos entrevistados, considerando assim humanizada a assistência em fisioterapia musculoesquelética (Tabela II).

Tabela 2. Relação fisioterapeuta-paciente

\begin{tabular}{|c|c|c|}
\hline Dimensões de atendimento & $\begin{array}{l}\mathrm{N}^{\circ} \text { (frequên- } \\
\text { cia })\end{array}$ & Porcentagem \\
\hline \multicolumn{3}{|l|}{ Dignidade } \\
\hline Positivo & 59 & $100 \%$ \\
\hline Negativo & 0 & $0 \%$ \\
\hline \multicolumn{3}{|l|}{ Comunicação } \\
\hline Positiva & 59 & $100 \%$ \\
\hline Negativa & 0 & $0 \%$ \\
\hline \multicolumn{3}{|l|}{ Autonomia } \\
\hline Positiva & 59 & $100 \%$ \\
\hline Negativa & 0 & $0 \%$ \\
\hline \multicolumn{3}{|l|}{ Confiabilidade } \\
\hline Positivo & 59 & $100 \%$ \\
\hline Negativo & 0 & $0 \%$ \\
\hline \multicolumn{3}{|l|}{ Garantia } \\
\hline Positivo & 59 & $100 \%$ \\
\hline Negativo & 0 & $0 \%$ \\
\hline \multicolumn{3}{|l|}{ Aspectos interpessoais } \\
\hline Positivo & 59 & $100 \%$ \\
\hline Negativo & 0 & $0 \%$ \\
\hline \multicolumn{3}{|l|}{ Empatia } \\
\hline Positivo & 59 & $100 \%$ \\
\hline Negativo & 0 & $0 \%$ \\
\hline \multicolumn{3}{|l|}{ Eficácia } \\
\hline Positivo & 59 & $100 \%$ \\
\hline Negativo & 0 & $0 \%$ \\
\hline \multicolumn{3}{|l|}{ Receptividade } \\
\hline Positivo & 59 & $100 \%$ \\
\hline Negativo & 0 & $0 \%$ \\
\hline
\end{tabular}




\section{DISCUSSÃO}

Foi considerado, diante dos pacientes interrogados, como humanizada a assistência fisioterapêutica, mostrando aprazimento com os serviços prestados pelos profissionais de fisioterapia que trabalham no centro de reabilitação. $O$ atendimento humanizado por esses profissionais foi ao encontro da dimensão humana do paciente, visto que se atribui respeito, dignidade e leva em consideração a autonomia do mesmo, exercendo papel fundamental na humanização da assistência. Notase a necessidade de o fisioterapeuta sempre acompanhar o paciente durante os atendimentos, incentivando a realização de suas tarefas. ${ }^{7}$

Lopes e Brito ${ }^{3}$ relatam que a idade pode interferir nas respostas, pois as necessidades e as expectativas das pessoas são diferentes de acordo com a fase de vida em que se encontram. Isso exige atenção redobrada e adequação das condutas às diferentes manifestações e comportamentos de cada paciente. O que se confirma no presente estudo, onde a grande maioria possui idade elevada, ou seja, requerem grande atenção e cuidado pelos profissionais.

Dentro do questionamento sobre a relação fisioterapeuta-paciente do presente estudo, todos os domínios receberam pontuações elevadas, o que orienta que o profissional de fisioterapia pode atuar com dignidade, autonomia, comunicação, confiança, empatia, eficácia, receptividade e confiança.

O item dignidade atingiu $100 \%$ das respostas na pesquisa, tornando a conduta baseada nos princípios éticos, tendo como objetivo o respeito à dignidade do ser humano e o cuidado com a sua saúde, comprometendose, ainda, com questões próprias da bioética, assim como rege o código profissional da fisioterapia. ${ }^{4}$

O quesito comunicação faz-se importante para que haja uma boa interação entre terapeuta-paciente, ouvindo cuidadosamente e atenciosamente, dispondose de tempo aos questionamentos realizados, mostrando que o paciente não é um simples objeto que necessita de atendimento, mas sim, um ser humano que precisa ser tratado como tal. ${ }^{1}$ No presente estudo, os indivíduos relataram que durante os atendimentos da fisioterapia, os profissionais sempre dialogavam com os pacientes, de modo que proporcionaram uma terapia mais aconchegante, na qual eles se sentiam mais à vontade.

O domínio da autonomia do questionário também obteve respostas satisfatórias, o que garantiu a liberdade para o indivíduo expressar sua opinião, tendo o livre arbítrio de decidir se aceita ou recusa o plano de tratamento proposto pelos fisioterapeutas, sendo importante para que o profissional aceite e respeite a decisão do paciente.

No próximo item, onde encontramos as respostas do questionário sobre o domínio da confiabilidade, os entrevistados a classificaram como humanizada, garantindo o cumprimento daquilo que foi prometido, assim como, ser apto a realizar o mesmo, suprindo as expectativas propostas aos indivíduos. Segundo Lopes e Brito $^{3}$, a garantia é um dos principais fatores para determinar a satisfação com a humanização da assistência de fisioterapia, pois pacientes esperam que o profissional de saúde gere uma situação de acolhimento que alivie ou amenize o sofrimento e que possa responder resolutamente ao seu problema de saúde.

Já no domínio dos aspectos interpessoais, as respostas positivas foram relacionadas com o respeito, cortesia, ânimo e interesse com que cada profissional fisioterapeuta proporciona a seu paciente, contribuindo dessa forma para um melhor ambiente de tratamento e resultados mais eficazes. A empatia que também se remete a esses aspectos, orienta-o a colocar-se um no lugar do outro, a fim de melhor compreender o que este outro vivencia, experimenta e sente, sendo uma importante característica destacada pelos fisioterapeutas, juntamente com a responsabilidade, a ética e a sensibilidade. ${ }^{8}$

O domínio da eficácia, com $100 \%$ de respostas positivas nessa pesquisa, proporcionou bons resultados aos pacientes que receberam alta, gerando assim melhoria da saúde do paciente. Um atendimento baseado na receptividade se faz cada vez mais importante e presente nesse meio, ganhando proporção significativa e bons resultados na relação profissional-paciente, fazendo dela algo mais humano, pois consiste em ser compreensivo e capaz de receber e transmitir opiniões, sem demonstrar insignificância e preconceito, respeitando o querer e o saber do outro, o que faz dele um ser mais humano.

Outros estudos também utilizam o mesmo 
instrumento para avaliar a relação terapeuta-paciente e todos eles encontram resultados positivos, classificando como humanizado o atendimento dos profissionais de fisioterapia. Lopes e Brito ${ }^{3}$ e Mondadori et $a l^{4}{ }^{4}$ apresentaram em seus estudos resultados positivos quanto ao grau de satisfação com a assistência da fisioterapia no período pós-alta da unidade de terapia intensiva adulto, da mesma forma que no estudo realizado por Zeni et al. ${ }^{5}$, onde os autores também obtiveram resultados positivos quanto à assistência oferecida pelos fisioterapeutas em unidade de terapia intensiva (UTI) neonatal e pediátrico. Já no estudo de Bomtempo e Taglietti ${ }^{6}$, as respostas positivas vieram dos pacientes de pós-operatório de cirurgia bariátrica.

Atitudes simples com o paciente podem humanizar o atendimento como, por exemplo, se preocupando com a sua dor, aproximar-se da família, explicar o que será feito pelo fisioterapeuta, prestar atenção na fala do paciente, preservar sua privacidade, possibilitar que ele participe de seu processo de cura, agindo com carinho, respeito e ética. ${ }^{9}$

Cabe ao profissional da reabilitação buscar alternativas para melhorar a assistência ao cliente, fundamentada não apenas na técnica, mas também em valores pessoais, apreendendo ainda a compreensão do verdadeiro significado e abrangência do cuidado humano, o que implica na necessidade de direcionar a assistência ao conforto físico associado à prestação de cuidados que visam amenizar a dor e o sofrimento. ${ }^{10}$

Nessa busca, o profissional pode contar com as políticas públicas de humanização, as quais se encontram longe da prática clínica pelos profissionais de reabilitação. A Política Nacional de Humanização ${ }^{11}$ preconiza a otimização do atendimento ao usuário, articulando a agenda multiprofissional em ações diagnósticas, terapêuticas que impliquem diferentes saberes e terapêuticas de reabilitação. Todavia, nessa pesquisa, os domínios do questionário vão ao encontro desses saberes, verificado pela totalidade das respostas positivas encontradas.

Outro aspecto importante que é evidente nessa pesquisa, é o modelo terapeuta paciente adotado, que no caso, englobou o modelo comunicacional/ biopsicossocial, garantido pelos domínios empregados no questionário. Nesse modelo, o paciente é visto e cuidado dentro de suas dimensões físicas e psicológicas, sendo incorporado os sentidos e significados no que marca a sua dimensão biopsicossocial. ${ }^{12}$ Essas características são aquelas que a Política Nacional de Humanização orienta, com intuito de fugir do modelo tradicional biomédico que os profissionais da saúde se encontram, pautado na mecanicidade e no princípio da causa-efeito. ${ }^{12}$

Diante do estudo proposto, foi possível evidenciar que não houve resultados negativos, todos os quesitos foram respondidos com $100 \%$ de positividade, o que evidenciou que os serviços e procedimentos fisioterapêuticos prestados são de excelente qualidade, definindo-os assim, como humanizados. A avaliação positiva dos quesitos dignidade, comunicação, autonomia, confiabilidade, garantia, aspectos interpessoais, empatia, eficácia e receptividade mostra que os profissionais fisioterapeutas utilizaram de abordagens as quais colocavam o paciente em enfoque, tendo em vista sempre o modelo biopsicossocial, fazendo disso algo humanizado. O fisioterapeuta deve procurar priorizar em seu atendimento a qualidade e a humanização, na qual o envolvimento do dia a dia deve acontecer de forma despercebida, sem fugir do contexto da humanização. ${ }^{13}$

Vale salientar que o estudo apresenta limitações, sendo que o questionário foi adaptado do ambiente hospitalar para o ambulatorial; o resultado de 100\% de positividade representa somente um serviço de saúde público/privado e que, portanto, não reflete necessariamente a satisfação com outros serviços de reabilitação. A entrevista face a face pode coibir as respostas pelo avaliador.

Novos estudos devem enfocar na validação de questionários, preferencialmente que discorram sobre o modelo terapeuta-paciente atual (ou o confronto com modelos antigos), bem como metodologias que aprofundem instrumentos, técnicas e recursos empregados pelos profissionais da reabilitação, além de estudos interdisciplinares na área da bioética, terapeuta paciente e humanização da assistência. 


\section{CONCLUSÃO}

Conclui-se que a conduta profissional adotada pelos fisioterapeutas atuantes em um serviço de reabilitação traumato-ortopédica foi classificada como humanizada pelos pacientes que necessitaram dessa assistência fisioterápica.

Conflitos de interesse: os autores declaram não haver conflitos de interesse.

\section{REFERÊNCIAS}

1. Silva ID, Silveira MFA. A humanização e a formação do profissional em fisioterapia. Ciên Saúde Coletiva. 2011;16(1):1535-44.

2. Machado NP, Nogueira LT. Avaliação da satisfação dos usuários de serviços de Fisioterapia. Rev Bras Fisiot. 2008;12(5):402.

3. Lopes MF, Brito ES. Humanização da assistência de fisioterapia: estudo com pacientes no período pósinternação em unidade de terapia intensiva. Rev Bras Ter Intensiva. 2009;21(3):285-286.

4. Mondadori AG, Moraes ZE, Taglietti M. Humanização da Assistência da Fisioterapia em Unidade Intensiva Pediátrica e Neonatal. Revista Assobrafir Ciência. 2016;7(3):296-298.

5. Zeni EM, Mondadori AG, Oliveira CCS, Wolf VLW, Taglietti M. Humanização da Fisioterapia em Unidade de Terapia Intensiva Adulto: estudo transversal. Revista Fisioter pesqui. 2016;23(3):294-300.

6. Bomtempo BG, Taglietti M. Humanização da assistência de fisioterapia no pós-operatório de cirurgia bariátrica. Revista Brasileira de Obesidade, Nutrição e Emagrecimento, 2017;11(66):479-485.

7. Almeida WA. A fisioterapia hospitalar e sua assistência humanizada [Monografia]. Campo Grande: PUC-MG Especialização em Fisioterapia Cardiopulmonar e Terapia Intensiva; 2015.

8. Costa SC, Figueireido MRB, Schaurich D. Humanização em unidade de terapia intensiva adulto (UTI): compreensões da equipe de enfermagem.
Interface - Comunic Saúde Educ. 2009;13(1):573577.

9. Silva MJP, Araujo MMT, Puggina ACG. Humanização em Unidades de Terapia Intensiva. Enfermagem em UTI: cuidando do paciente crítico. Barueri: Manole, 2010.

10. Caetano JA, Andrade LM, Soares E, Ponter RM. Cuidado humanizado em terapia intensiva: um estado reflexivo. Revista de Enfermagem. 2007;11(2):329.

11. Brasil. Ministério da Saúde. Núcleo Técnico da Política Nacional de Humanização. HumanizaSUS: política nacional de humanização: documento base para gestores e trabalhadores do SUS / Ministério da Saúde, Secretaria-Executiva - 2. Ed. - Brasília: Ministério da Saúde, 2004.

12. Lima PM, Oliveira ACM. Relação profissionalpaciente: a busca pela humanização nos serviços de saúde. Revista Fluminense de Odontologia. 2012;1(37):47-50.

13. Warpechowski TR. A possibilidade de humanizar o Atendimento Fisioterapêutico. In: Salão do Conhecimento, 2015, Ijuí. XX Jornada de Pesquisa, 2015. 
Apêndice I. Questionário: humanização da assistência de fisioterapia em serviço de referência em reabilitação.

\section{A) Características sociodemográficas}

01. Sexo:
1. ( ) $\mathrm{F}$
2. ( ) M

02. Idade:

03. Estado civil:

1. ( )Solteiro

2. ( ) Casado

04. Escolaridade:

1. ( ) Sem escolaridade formal

2. ( ) Ensino fundamental:

2.1 ( ) Incompleto 2.2 ( ) Completo

3. ( ) Ensino médio:

3.1 ( )Incompleto 3.2 ( ) Completo

4. ( ) Ensino superior 4.1 ( ) Incompleto 4.2 ( ) Completo

05. Renda (Salários mínimos):

1. ( ) 0 a 3

2. ( ) $>3$

06. Classe de diagnóstico primário:

1. ( ) Clínico

1.1. ( ) Respiratório

1.2. ( ) Cardiovascular

1.3. ( ) Neurológico

1.4. ( ) Ortopédico/Traumatológico

1.5. ( ) Aquático

1.6. ( ) Pediátrico

1.7. ( ) Dermatofuncional

1.8. ( ) Prótese e Órtese

1.9. ( ) Outros:

\section{B) Relação fisioterapeuta-paciente}

01. Dignidade: "Ter um atendimento digno, atencioso e respeitoso. Ser identificado e tratado pelo nome. Poder identificar os fisioterapeutas envolvidos na sua assistência. Ter assegurada sua privacidade, individualidade e respeito aos seus valores éticos e culturais."

\section{1.( ) Positiva \\ 2. ( ) Negativa}

02. Comunicação: "Receber informações claras, objetivas e compreensiveis. Ser ouvido cuidadosamente pelo fisioterapeuta, dispondo de tempo suficiente para esclarecer todas as dúvidas."

\section{1.( ) Positiva \\ 2. ( ) Negativa}

03. Autonomia: "Ter informação sobre as opções e alternativas de tratamento e permissão para tomar decisões sobre o tipo de tratamento, depois de discutir com o fisioterapeuta. Poder recusar o tratamento."
1.( ) Positiva
2. ( ) Negativa

04. Confiabilidade: "Os fisioterapeutas cumprirem o que prometem e estarem babilitados para realizar o serviço."
1.( ) Positiva
2. ( ) Negativa

05. Garantia: "Serviço de fisioterapia aliando práticas resolutivas e babilidade de seu desempenbo."
1.( ) Positiva
2. ( ) Negativa

06. Aspectos Interpessoais: "Modo com que cada fisioterapeuta interage pessoalmente com os pacientes, ou seja, respeito, cortesia, interesse, ânimo.”

1. ( ) Positiva 2. ( ) Negativa

07. Empatia: "Fisioterapeutas com habilidade de imaginar-se no lugar do paciente e oferecer uma assistência individualizada."
1.( ) Positiva
2. ( ) Negativa

08. Eficácia: "Atendimento resolutivo baseado em critérios de risco, resultando em melhoria ou manutenção da saúde."
1.( ) Positiva
2. ( ) Negativa
09. Receptividade: "Prontidão em ajudar a responder as necessidades dos pacientes."

1.( ) Positiva 2. ( ) Negativa 